\title{
Exchange instability of the two-dimensional electron gas in semiconductor quantum wells
}

\author{
A.R. Goñi ${ }^{1}$, U. Haboeck ${ }^{1}$, C. Thomsen ${ }^{1}$, K. Eberl ${ }^{2}$, F.A. Reboredo ${ }^{3}$, C.R. Proetto ${ }^{3}$, and \\ F. Guinea ${ }^{4}$ \\ ${ }^{1}$ Institut für Festkörperphysik, Technische \\ Universität Berlin, Hardenbergstr. 36, 10623 Berlin, Germany \\ ${ }^{2}$ MPI für Festkörperforschung, Heisenbergstr. 1, \\ 70569 Stuttgart, Germany \\ ${ }^{3}$ Comisión Nacional de Energía Atómica, Centro Atómico \\ Bariloche, 8400 S.C. de Bariloche, Argentina \\ ${ }^{4}$ Instituto de Ciencias de Materiales (CSIC), Cantoblanco, \\ 28049 Madrid, Spain
}

\begin{abstract}
A two-dimensional (2D) electron gas formed in a modulation-doped GaAs/AlGaAs single quantum well undergoes a first-order transition when the first excited subband is occupied with electrons, as the Fermi level is tuned into resonance with the excited subband by applying a dc voltage. Direct evidence for this effect is obtained from low-temperature photoluminescence spectra which display the sudden renormalization of the intersubband energy $E_{01}$ upon the abrupt occupation of the first excited subband. Calculations within density-functional theory, which treat the $2 D$ exchange potential exactly, show that this thermodynamical instability of the electron system is mainly driven by intersubband terms of the exchange Coulomb interaction. From temperature-dependent measurements the existence of a critical point
\end{abstract}


at $T_{c}=35 \pm 5 \mathrm{~K}$ is inferred.

PACS: 71.30.+h, 78.55.-m, 71.10.-w, 73.21.Fg

Typeset using REVTEX 
Exchange effects are a fundamental manifestation of electron-electron interactions in many-particle systems like high-mobility two-dimensional (2D) electron gases which form in modulation-doped semiconductor quantum well structures. Exchange-correlation interactions are at the origin of a vast variety of fascinating quantum phenomena in solids [1], for example, spin excitations, magnetic ordering, excitonic binding and band-gap renormalization among others. The latter, for instance, is apparent in semiconductor heterostructures as a reduction of gap energies when the corresponding band states become populated with carriers either by varying doping levels [2,3] or under intense photoexcitation [4]. This effect is explained as arising from exchange-correlation corrections due to the presence of free carriers in the system [5, 6].

Electronic correlations dictate the behavior of dilute electron gases of reduced dimensions particularly at very low densities. For instance, the occurrence of a metal-insulator transition (MIT) in two dimensions at zero magnetic field [7,8] is in open contrast with the prediction of scaling theory that a noninteracting 2D electron or hole system becomes localized at low temperatures for any degree of disorder [9]. Recently, other thermodynamic properties of $2 \mathrm{D}$ electron gases like the chemical potential and its density derivative, the compressibility, have been found to exhibit anomalous behavior across the metal-insulator transition deviating from what is expected within Hartree-Fock theory [10]. In spite of the universalities detected at the MIT, for example in the temperature dependence of the resistivity [11, this transition does not reveal a purely intrinsic property of interactions in low-dimensional systems since disorder plays the crucial role. On the contrary, localization effects can be widely suppressed in double-layer electron gases, in which the electron gas in one quantum well is used for the complete screening of the disorder potential of the ionized donors [12]. Using a capacitance technique Eisenstein et al. [12] were able to show that at low densities but without magnetic field the compressibility of a high-mobility 2D electron gas becomes negative owing mainly to exchange interactions. This leads to a thermodynamical instability of the electron system. Furthermore, exchange effects might induce other types of instabilities in double quantum wells such as a (controversial) bilayer-to-monolayer transition [13] or even one towards a magnetic 
ground state [14]. The exchange interaction also gives rise to first-order transitions in diluted magnetic semiconductors [15].

In this Letter we show that at low temperature and zero magnetic field a 2D electron gas formed in a single GaAs quantum well undergoes a first-order phase transition, as the first excited electron subband becomes populated with electrons by raising the Fermi level with a gate voltage. The evidence is found in the sudden and abrupt renormalization of the energy of the first excited subband, as determined from photoluminescence and inelastic light scattering measurements. Self-consistent density-functional calculations with exact exchange potential for a $2 \mathrm{D}$ electron system reveal that this transition is driven by intersubband exchange interactions which provide a feedback mechanism for charge transfer into the excited subband. Experiments performed at different temperatures indicate the existence of a critical point for this transition at around $35 \pm 5 \mathrm{~K}$.

The sample consists of a modulation-doped $245 \AA$-wide GaAs single quantum well (SQW) with $\mathrm{Al}_{0.33} \mathrm{Ga}_{0.67} \mathrm{As}$ barriers grown by molecular-beam epitaxy. The growth sequence is given elsewhere [8]. Without bias only the lowest subband is occupied with electrons with Fermi energy $E_{F} \approx 25 \mathrm{meV}$. The energy separation to the second subband is $E_{01} \approx 28 \mathrm{meV}$. The electron gas is contacted from the surface by In alloying in order to apply a dc bias up to $30 \mathrm{~V}$ between it and a metallic back contact. Photoluminescence (PL) and inelastic light scattering spectra were excited with a tunable Ti:sapphire laser and recorded with optical multichannel detection.

The effect of the applied voltage is an increase of the electron density in the quantum well shifting the Fermi level in the structure towards degeneracy with the bottom of the first excited subband. Figure 1 shows some PL spectra recorded at $7 \mathrm{~K}$ and for different bias. The low-energy emission line $E_{0}$ corresponds to recombination processes between the lowest electron and heavy-hole subbands (with subband index 0). The peak labeled as $E_{1}$ is associated with optical transitions between the first excited electron subband and the hole ground state, which become dipole allowed due to the lack of inversion symmetry of the triangular potential in the doped well. The peak at $1.515 \mathrm{eV}$ originates from near 
bandgap emission of the GaAs buffer layer. At high voltages a high-energy cutoff at the Fermi energy $E_{F}^{\prime}=E_{F}\left(1+m_{e} / m_{h}\right)$ is clearly apparent from the PL spectra. The factor containing the ratio of electron and hole effective masses accounts for the curvature of the valence band. With increasing bias the Fermi energy increases leading to a population of the first excited subband. An important result concerns the strong redshift of the subband energy $E_{1}$ by about $11 \mathrm{meV}$. Moreover, the occupation of the second subband proceeds abruptly at the voltage for which the Fermi level reaches its bottom. This reduction of $E_{1}$ is a consequence of bandgap renormalization effects due to exchange-correlation corrections in the presence of free carriers [5, 6].

The dependence on bias of the electron densities $n_{0}$ and $n_{1}$ of the ground state and first excited subband, respectively, as well as the intersubband energy $E_{01}$ has been determined from a quantitative analysis of PL line shapes, as described elsewhere [3,8]. The values for $n_{0}, n_{1}$ and $E_{01}$ obtained at $10 \mathrm{~K}$ are plotted in Fig. 2 as a function of the Fermi level referred to the top of the valence band. Below $40 \mathrm{~K}$ when the Fermi level equals $E_{1}$ the electron density $n_{1}$ jumps from zero to a finite value ranging from 3 to $8 \times 10^{10}$ $\mathrm{cm}^{-2}$ depending on temperature. The electron density of the lowest subband, in contrast, increases slightly but smoothly with voltage. Simultaneously with the abrupt filling of the second subband, a sudden reduction of the intersubband energy $E_{01}$ by about $3.5 \mathrm{meV}$ is observed [16]. Bandgap renormalization acts as a feedback mechanism for subband filling leading to a sudden population of the excited electron subband and, in addition, causing the pronounced decrease of the intersubband spacing in the well. We point out that PL provides a means for the determination of the Fermi level which is independent of the quality of the contacts or the way in which charge flows into the quantum well. We thus rule out spurious charging effects as cause of the observed jumps in 2D density and intersubband spacing.

The observed discontinuities in electron density and gap renormalization upon occupation of the first excited subband point to a thermodynamical instability of the 2D electron gas. In order to enlighten this fundamental issue, we have performed self-consistent cal- 
culations of the subband structure and level occupation of the single quantum well within the formalism of density functional theory (DFT). Our calculation differs from the standard local-density approximation (LDA) in two mayor points. The exchange interaction is treated exactly [17] for a quasi-2D electron system and the number of particles is not fixed but is allowed to change. Mathematically, the exchange potential is calculated according to

$$
\begin{aligned}
V_{x}(\mathbf{r})= & \frac{\partial E_{x}}{\partial \rho(\mathbf{r})}=\int \mathbf{d r}^{\prime}\left\{\sum_{v k} \int \mathbf{d r}^{\prime \prime}\left[\frac{\partial E_{x}}{\partial \phi_{n \mathbf{k}}\left(\mathbf{r}^{\prime}\right)} \frac{\partial \phi_{n \mathbf{k}}\left(\mathbf{r}^{\prime}\right)}{\partial V_{K S}\left(\mathbf{r}^{\prime \prime}\right)}+c . c .\right]+\right. \\
& \left.+\sum_{v}\left[\frac{\partial E_{x}}{\partial k_{F}^{v}} \frac{\partial k_{F}^{v}}{\partial V_{K S}\left(\mathbf{r}^{\prime \prime}\right)}\right]\right\} \frac{\partial V_{K S}\left(\mathbf{r}^{\prime \prime}\right)}{\partial \rho(\mathbf{r})},
\end{aligned}
$$

where $E_{x}$ is the exact exchange energy, $\rho(\mathbf{r})$ is the electron density, $V_{K S}(\mathbf{r})=V_{e x}(\mathbf{r})+$ $V_{H}(\mathbf{r})+V_{x}(\mathbf{r})+V_{c}(\mathbf{r})$ is the Kohn-Sham potential given as sum of the external, Hartree, exchange and correlation potentials, respectively, and $\phi_{n \mathbf{k}}(\mathbf{r}) \propto e^{i \mathbf{k} . \rho} \cdot \xi_{n}(z)$ is the wave function of an electron in the quantum well characterized by the envelope function $\xi_{n}(z)$ in the direction of confinement $z$. The fist term in Eq. (11) represents derivatives of $E_{x}$ with respect to the shape of the wave functions [17], whereas the second one accounts for the variation of the exchange energy with the occupations.

Integration in the $(x, y)$ plane yields (in atomic units)

$$
\begin{gathered}
V_{x}(z)=\frac{1}{2}\left\{\sum_{v v^{\prime} n} I_{1}\left(v, v^{\prime}, n\right) \int d z^{\prime} \frac{\xi_{v}\left(z^{\prime}\right) \xi_{n}\left(z^{\prime}\right)}{\varepsilon_{v}-\varepsilon_{n}} \chi^{-1}\left(z, z^{\prime}\right)+\right. \\
\left.+\sum_{v v^{\prime}} I_{2}\left(v, v^{\prime}\right) \int d z^{\prime}\left|\xi_{v}\left(z^{\prime}\right)\right|^{2} \chi^{-1}\left(z, z^{\prime}\right)\right\} \\
I_{1}\left(v, v^{\prime}, n\right)=k_{F}^{v} k_{F}^{v^{\prime}} \int \frac{d \rho}{\rho} J_{1}\left(k_{F}^{v} \rho\right) J_{1}\left(k_{F}^{v^{\prime}} \rho\right) \int d z_{1} d z_{2} \frac{\xi_{v}\left(z_{1}\right) \xi_{n}\left(z_{2}\right) \xi_{v^{\prime}}\left(z_{1}\right) \xi_{v^{\prime}}\left(z_{2}\right)}{\sqrt{\rho^{2}+\left(z_{1}-z_{2}\right)^{2}}} \\
I_{2}\left(v, v^{\prime}\right)=k_{F}^{v^{\prime}} \int d \rho J_{0}\left(k_{F}^{v} \rho\right) J_{1}\left(k_{F}^{v^{\prime}} \rho\right) \int d z_{1} d z_{2} \frac{\xi_{v}\left(z_{1}\right) \xi_{v}\left(z_{2}\right) \xi_{v^{\prime}}\left(z_{1}\right) \xi_{v^{\prime}}\left(z_{2}\right)}{\sqrt{\rho^{2}+\left(z_{1}-z_{2}\right)^{2}}}
\end{gathered}
$$


where the operator $\chi\left(z, z^{\prime}\right)=\partial \rho(z) / \partial V_{K S}\left(z^{\prime}\right)$ is related to the Linhard susceptibility of the $2 \mathrm{D}$ gas in the $\mathrm{T} \longrightarrow 0$ limit. In Eq. (2), $v$ and $v^{\prime}$ sum only occupied subbands, while the index $n$ runs over all subbands (with exception of $n=v$ ). $J_{i}(z)$ stands for the Bessel function of order $i, \rho$ is the in-plane coordinate vector and $k_{F}^{v}$ and $k_{F}^{v^{\prime}}$ are the Fermi wave vectors of the occupied subbands.

The striking and novel result of our theory is that the exchange potential $V_{x}(z)$ changes discontinuously every time a subband becomes occupied with electrons. For our experimental situation, i.e. $v^{\prime}=0$ and $v=1$, the second term in Eq. (2) is finite even for $k_{F}^{v=1} \longrightarrow 0^{+}$because $I_{2}(1,0) \neq 0$, whereas the term containing $I_{1}(1,0, n)$ goes to zero. The operator $\chi^{-1}\left(z, z^{\prime}\right)$ also presents a discontinuity as soon as $k_{F}^{v=1}>0$. The self-consistent solution of the Kohn-Sham equations with $V_{x}(z)$ given by Eq. (2), in fact, exhibits an abrupt jump from a phase with one subband occupied to a phase with two occupied subbands when the Fermi level equals $E_{1}$. We obtain at occupation of subband 1 a sudden reduction of $E_{01}$ by about $2.5 \mathrm{meV}$ for an initial value of $26.5 \mathrm{meV}$, which is in good quantitative agreement with the experimental observation. This is a clear signature of an exchange-driven first-order phase transition. This drop of the intersubband spacing is due to an effective level repulsion between the first excited subband and the Fermi energy due to exchange interaction. Due to intersubband exchange terms it is more favorable for the 2D electron gas to transfer a macroscopic amount of charge into the first excited subband upon occupation, thus, leading to the sudden collapse of the subband spacing $E_{01}$. Details of the theory and the self-consistent calculations will be given elsewhere [18].

Further evidence for the first-order character of the exchange instability in electron gases by occupation of higher subbands can be gained from PL measurements at different temperatures. Figure 3 displays several isothermal curves representing the variation of the Fermi level and the density of the first excited subband. The isotherms fall into two classes according as the temperature lies below or above a critical temperature $T_{c}=35 \pm 5$ K. Below $T_{c}$ the $2 \mathrm{D}$ electron gas can exist in two states with and without population of 
the first excited subband; its occupation proceeds abruptly due to the exchange instability. Above the critical temperature, on the contrary, the discontinuity in the density $n_{1}$ disappears and the system evolves continuously from one phase to another. In addition, evidence of a phase mix revealing a spatial inhomogeneity of the electron gas is obtained from luminescence. At the instability and with fixed voltage PL spectra display a multiple-peak time-varying structure at energies around $E_{1}$. Each peak of this feature is assigned to PL emission at $E_{1}$ arising from different regions within the laser spot with slightly different electron density.

In conclusion, we have found that a 2D electron gas formed in a modulation-doped GaAs single quantum well undergoes a first-order phase transition when the first excited subband becomes populated by raising the Fermi level in the structure using a dc voltage. The signature of this transition is seen in the abrupt renormalization of the subband energy and the associated jump in electron density upon occupation of the excited subband. Furthermore, we have determined from the isotherms a critical temperature of about $35 \mathrm{~K}$ for the transition from the phase without to that with finite occupation of the excited subband. Self-consistent calculations within density-functional theory, which treat the exchange interaction in the 2D system exactly, show that such thermodynamical instability of the electron gas is mainly induced by intersubband exchange terms of the Coulomb interaction. This instability corresponds, for instance, to the same universal class of liquid-vapor phase transitions. In spite of the importance of thermodynamical fluctuations in two dimensions, they are not sufficiently large in order to prevent the exchange-driven transition from being of first order. In this way, we have provided further insight into the issue of the many-body behavior of high-mobility electron gases in 2D arising from exchange interactions between correlated electrons in different subbands of semiconductor quantum wells.

This work is supported in part by the Deutsche Forschungsgemeinschaft in the framework of Sfb 296. F. A. R. and C. R. P. acknowledge support from CONICET of Argentina. F.G. acknowledges financial support from CICyT (Spain), through grant PB96-0875. 


\section{REFERENCES}

[1] G.D. Mahan, Many-particle Physics (Plenum, New York, 1981).

[2] C. Delalande, G. Bastard, J. Orgonasi, J.A. Brum, H.W. Liu, M. Voos, G. Weimann, and W. Schlapp, Phys. Rev. Lett. 59, 2690 (1987).

[3] A.R. Goñi, M. Danckwerts, U. Haboeck, K. Eberl, and C. Thomsen, phys. stat. sol. (b) 215, 347 (1999).

[4] R. Cingolani, H. Kalt, and K. Ploog, Phys. Rev. B 42, R7655 (1990) and references therein.

[5] D.A. Kleinman and R.C. Miller, Phys. Rev. B 32, 2266 (1985).

[6] S. Das Sarma, R. Jalabert, and S.-R. E. Yang, Phys. Rev. B 41, 8288 (1990).

[7] S.V. Kravchenko, G.V. Kravchenko, J.E. Furneaux, V.M. Pudalov, and M. D'Iorio, Phys. Rev. B 50, 8039 (1994); Y. Hanein, U. Meirav, D. Shahar, C.C. Li, D.C. Tsui, and H. Shtrikman, Phys. Rev. Lett. 80, 1288 (1998).

[8] S. Ernst, A.R. Goñi, K. Syassen, and K. Eberl, Phys. Rev. Lett. 72, 4029 (1994).

[9] E. Abrahams, P.W. Anderson, D.C. Licciardello, and T.V. Ramakrishnan, Phys. Rev. Lett. 42, 673 (1979).

[10] S. Ilani, A. Yacoby, D. Mahalu, and H. Shtrikman, Phys. Rev. Lett. 84, 3133 (2000).

[11] S.V. Kravchenko, W.E. Mason, G.E. Bowker, J.E. Furneaux, V.M. Pudalov, and M. D'Iorio, Phys. Rev. B 51, 7038 (1995).

[12] J.P. Eisenstein, L.N. Pfeiffer, and K.W. West, Phys. Rev. Lett. 68, 674 (1992); ibid. Phys. Rev. B 50, 1760 (1994).

[13] L. Zheng, M.W. Ortalano, and S. Das Sarma, Phys. Rev. B 55, 4506 (1997); F.A. Reboredo and C.R. Proetto, Phys. Rev. B 58, 7450 (1998); S. Das Sarma, M.W. Ortalano, and L. Zheng, Phys. Rev. B 58, 7453 (1998). 
[14] F. A. Reboredo and C. R. Proetto, Phys. Rev. Lett. 82, 1887 (1997).

[15] L. Brey and F. Guinea, Phys. Rev. Lett. 85, 2384 (2000).

[16] We note that the same dependence of $E_{01}$ on bias has been determined from inelastic light scattering by elementary excitations of the 2D gas associated with electronic transitions between subband 0 and 1 .

[17] M. Städele, M. Moukara, J. A. Majewski, P. Vogl, and A. Görling, Phys. Rev. B 59, 10031 (1999).

[18] F. A. Reboredo and C. R. Proetto, in preparation. 


\section{FIGURES}

FIG. 1. Photoluminescence spectra of a modulation-doped $25 \mathrm{~nm}$ wide single quantum well at $7 \mathrm{~K}$ and for different gate voltages. The position of bandgaps and Fermi energy is indicated. The peak at $1.515 \mathrm{eV}$ arises from bulk GaAs luminescence.

FIG. 2. Dependence on gate voltage of (a) the electron densities $n_{0}$ and $n_{1}$ of the lowest and the first excited subband, respectively, and (b) of the intersubband spacing $E_{01}$, as determined from PL spectra at $10 \mathrm{~K}$. The curves are a guide to the eye.

FIG. 3. Measured isothermal curves of Fermi level versus electron density of the first excited subband for the 2D electron gas of the modulation-doped GaAs single quantum well. Above 30 $\mathrm{K}$ the discontinuity in $n_{1}$ is no longer observed (see text). 


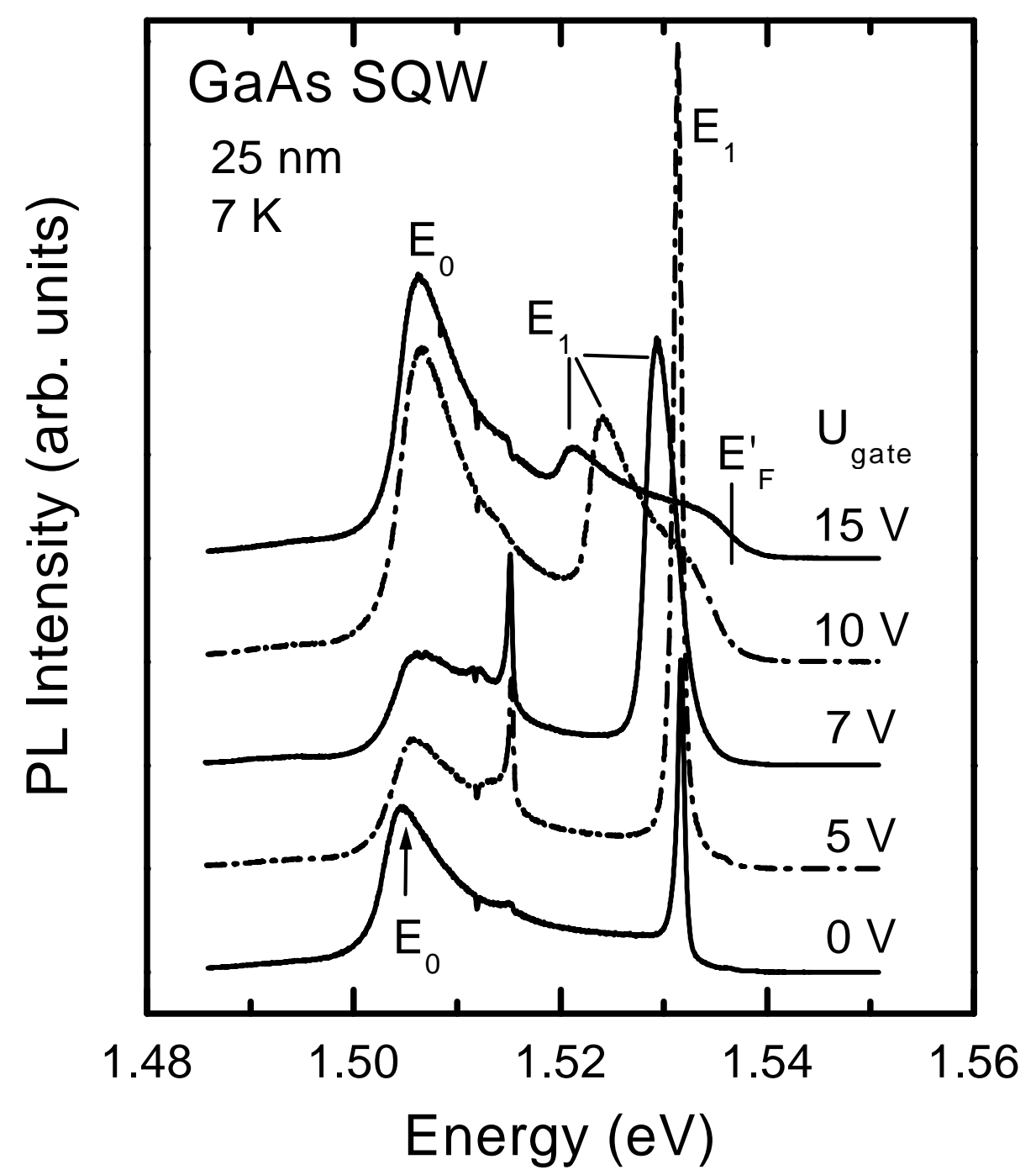

Fig. 1

Goñi et al. 


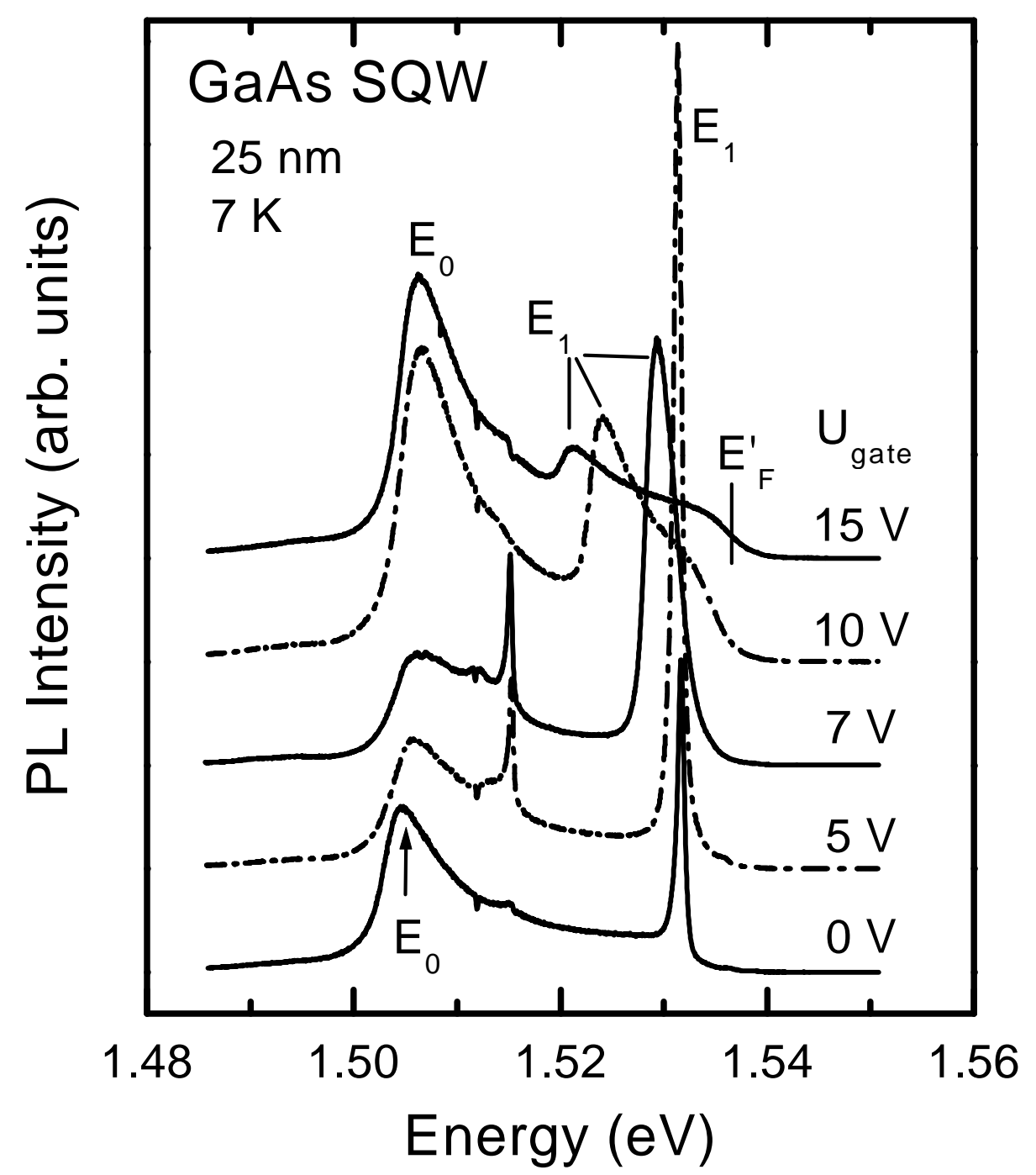

Fig. 1

Goñi et al. 


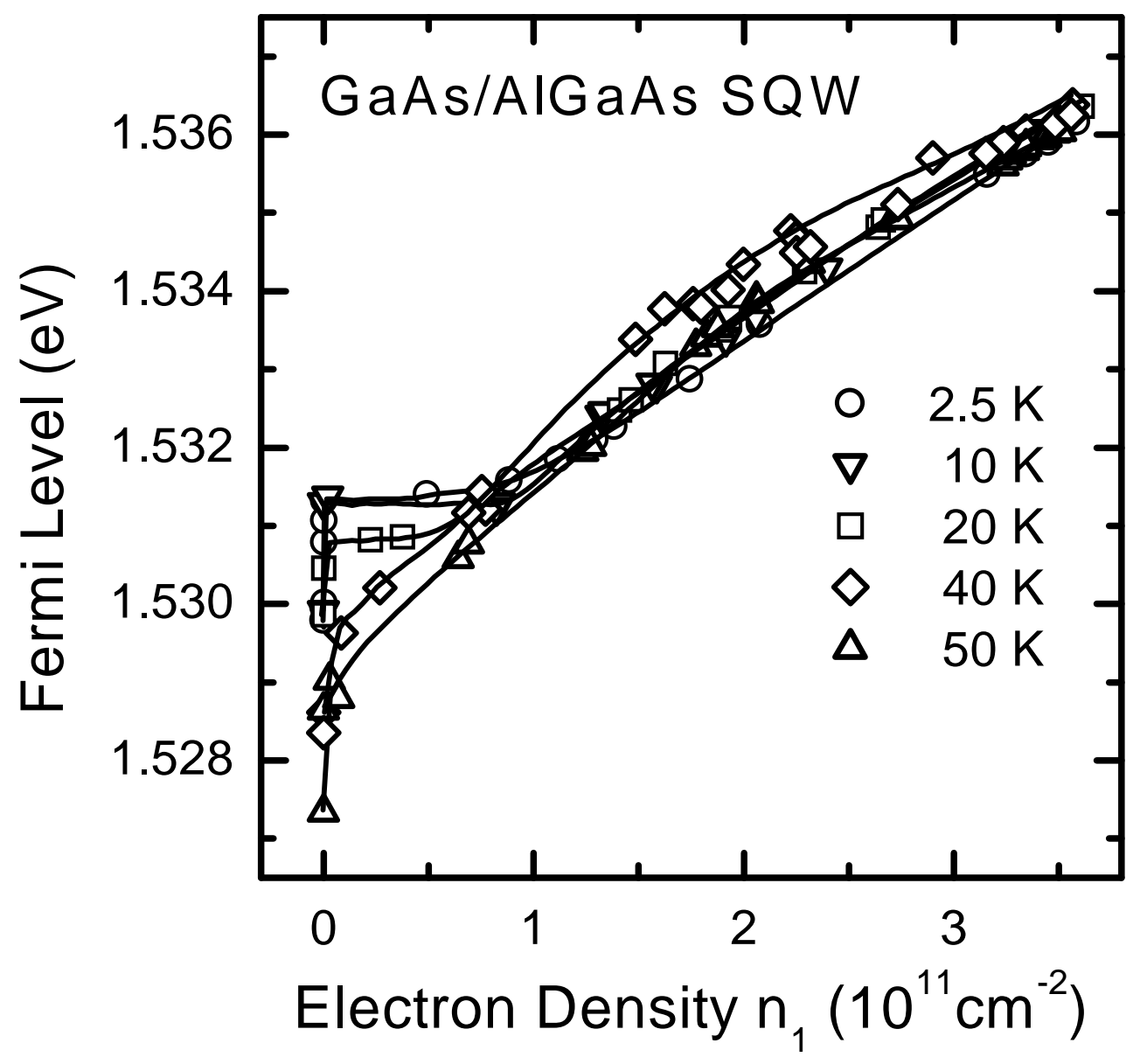

Fig. 3

Goñi et al. 